\title{
CAPACITANCE MEASUREMENTS FOR SUBCELL CHARACTERIZATION IN MULTIJUNCTION SOLAR CELLS
}

\author{
Carmen M. Ruiz ${ }^{1,2}$, Ignacio Rey-Stolle ${ }^{1}$, Iván García ${ }^{1}$, Enrique Barrigón ${ }^{1}$, Pilar Espinet ${ }^{1}$, V. Bermúdez ${ }^{3}$, Carlos Algora $^{1}$ \\ ${ }^{1}$ Instituto de Energía Solar - Universidad Politécnica de Madrid ETSI de Telecomunicación, Avda. Complutense s/n \\ 28040 Madrid. Spain \\ ${ }^{2}$ Instituto de Ciencia de Materiales de Sevilla (ICMSE) (CSIC-Univ. Sevilla) Avda. Américo Vespucio 49. \\ 41092 Sevilla. Spain \\ ${ }^{3}$ NEXCIS Photovoltaic Technology, 190 Av Celestin Coq 13190, Rousset, France
}

\begin{abstract}
On this paper we present an alternative way to analyze de electronic properties of each subcell from the complete device. By illuminating the cell with light sources which energy is near one of the subcell bandgaps, it is possible to "erase" the presence of such subcell on the CV curve. The main advantages of this technique are that it is not destructive, it can be measured on the complete cell so can be easily implemented as a diagnostic technique for controlling electronic deviations.
\end{abstract}

\section{INTRODUCTION}

There is a lack of characterization techniques regarding the whole structure on a multijunction solar cell. Although this kind of structure is the most direct way to obtain high efficiency cells, the catalogue of techniques available to perform diagnostics on the whole device is very scarce. For instance Quantum Efficiency can be used to discriminate the subcells by overstimulating the junctions not to be measured [1-3]. The polarization of the stack under reverse bias has been also proposed to measure the short-circuit current produced by each subcell in the multijunction stack under a given spectrum [4]. Also, spectroscopic electroluminescence produced by a GalnP/GalnAs/Ge triple-junction solar cell at different injection currents can be used for recovering the individual I-V curve of each subcell (and diode quality factors) [5] using the reciprocity theorem between electroluminescent emission and external quantum efficiency of solar cells [6].

On the other hand, capacitance based techniques and in particular capacitance-voltage (CV) curves have been widely used in photovoltaics [7-9]. The possibility of routine measurements over the finished device allows obtaining very useful information about the diode behavior under working conditions [10]. Nevertheless, these measurements have been mainly performed on single diodes and the knowledge for understanding the data obtained is still very small. The main problem is how to deal with a signal composed by different contributions of each diode which are not easy to deconvolute. On a previous paper [11] we have shown the possibility of measuring CV curves on III-V multijunction cells, and have discussed qualitatively the information that can be extracted.
On this work we present the possibility of tuning the CV measurements to one of the subcells, allowing the extraction of information of the diode on the finished cell.

\section{MODEL}

The inverse of the total capacitance of the whole multijunction solar cell is the sum of the inverses of the capacitance of each subcell. (Eq. 1). Therefore, the smaller the capacitance of a given subcell is, the larger is its influence:

$$
\frac{1}{C_{T o t}}=\sum_{i} \frac{1}{C_{i}}
$$

In addition, as indicated in Eq. 2, the capacitance of an asymmetric pn junction under reverse bias -as is typically the case in solar cells- is proportional to the square root of the charge density on the lightly doped side (i.e. typically the base). Thus, if somehow we are able to increase this charge density in the base, the capacitance signal of the associated junction will increase. Thereby, if we are able to artificially increase the capacitance of a subcell without varying the capacitance of the other subcells we would decrease its contribution on the total capacitance and eventually, we could "erase" this signal, allowing the analysis of the other subcells.

$$
C=\sqrt{\frac{K \cdot N_{a}}{\left(V-V_{b i}\right)}}
$$

Being $\mathrm{N}_{\mathrm{A}}$ the acceptor concentration in the base of the solar cell, $V_{b i}$ the built-in voltage, $V$ the value of the voltage and $\mathrm{K}$ a constant that includes the dielectric constant.

Near bandgap illumination allows to generate a large quantity of free carriers within the base and space charge region of a junction without altering the population in the rest of the device. For this, we propose illuminating the multijunction solar cell with an intense light with energy close to the bandgap of a given subcell in order to highly increase the charge density in the corresponding base. This way, the light will be mainly absorbed on the targeted subcell and the rest of the junctions will remain unaltered so we can fulfill the requirements previously described. 


\section{EXPERIMENTAL}

In order to test the predicted dependence for the capacitance on the incident light, dual-junction (DJ) GalnP/GaAs, and triple-junction (TJ), GalnP/GaAs/Ge solar cells were grown by MOPVE as it has been described elsewhere [12,13]. After encapsulation, Capacitance-Voltage (CV) measurements were taken as a function of temperature. For this, a HP 4284 LCR meter was coupled to a Janis 810 cryostat. A 320 Lakeshore temperature controller allowed us to change the temperature in the range of 260-370K. Laser diodes were used as variable high intensity light sources. The wavelength used for stimulating the GaAs diode $\left(\lambda_{\text {gap }}=\right.$ $870 \mathrm{~nm}$ ) was $814 \mathrm{~nm}$, while wavelength chosen for stimulating the GalnP subcell ( $\lambda_{\text {gap }}=680 \mathrm{~nm}$ ) was $658 \mathrm{~nm}$. In both cases, the laser intensity was variable, permitting us to perform measurements at different levels of saturation.

\section{RESULTS}

\section{Dual-junction solar cell}

For the GalnP/GaAs solar cell we have alternatively illuminated the same device with the $658 \mathrm{~nm}$ laser and with the $814 \mathrm{~nm}$ laser, applying different laser intensities. As the intensity of the laser is increased, the population in the spacedcharged region (SRC) increases on the corresponding subcell. For certain intensity, the subcell saturates and his contribution disappears of the general CV curve.

\section{Curves obtained with red light illumination}

In figure 1 the variation of the CV curve as a function of the light intensity is plotted for a monochromatic light of $654 \mathrm{~nm}$.

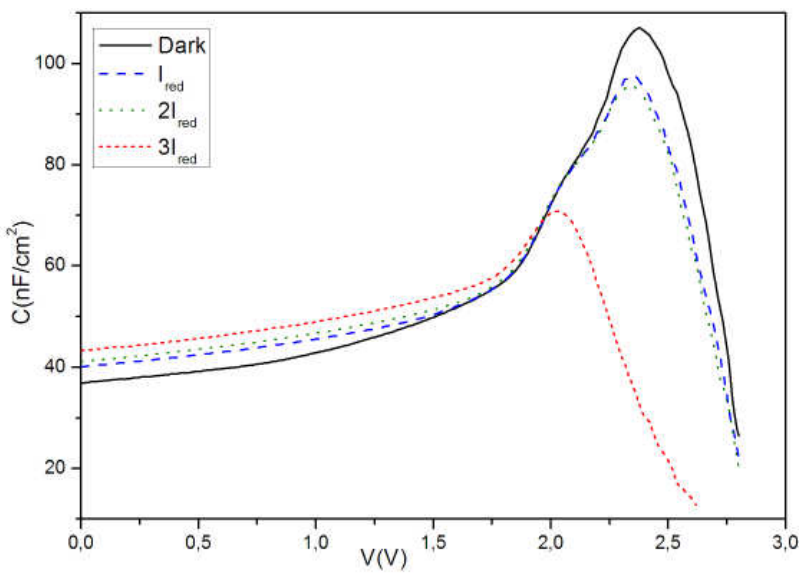

Figure $1 \mathrm{CV}$ curves for a DJ solar cell under red illumination for different laser intensities.
On this figure, we can clearly distinguish three different situations: (i) the curve measured under dark (solid line),(ii) the curve at junction saturation (dotted line), and (iii) intermediate states with increasing levels of light intensity (dashed lines).. The dark curve is like the curves obtained previously for DJ solar cells [11], where the capacitance contribution of both cells is present, and it is not possible to clearly separate each one- As the light intensity increases, , the form and the magnitude of the associated CV curve vary, with the maximum peak shifting to lower bias values and the capacitance maximum decreasing .

On the other hand, for the equilibrium state $\left(V_{\text {bias }}=0\right)$, the capacitance increases as the light intensity augments. This can be explained with eq. 1 If we increase the value of one of the capacitances (as we inject charges on the GalnP SCR) while the other subcell capacitance remains virtually constant, the total capacitance of the whole device will tend to the values of the constant one.

Finally, the dotted curve corresponds to the saturation of the GalnP junction. For larger laser intensities ( $>3 \mathrm{I}_{\text {red }}$ ), not significant variations can be found. Under this situation, the junction behaves as a constant capacitor, i.e. its capacitance value does not vary with the applied voltage. So the information on this curve corresponds mainly to the GaAs subcell. The form of the curve does not indicate any other contributions but this of one junction. We can calculate the net doping density of the base on the GaAs subcell, applying the Mott-Schottky equation [14]. The value for this cell is $N_{G a A s}=10^{17} \mathrm{~cm}^{-3}$, which is in good accordance with the growth conditions [12].

\section{Curves obtained with infrared illumination}

The next step is to be able to analyse also the GalnP subcell. For this we use the $814 \mathrm{~nm}$ laser and start to increase the light intensity. Curves obtained are plotted on figure 2.

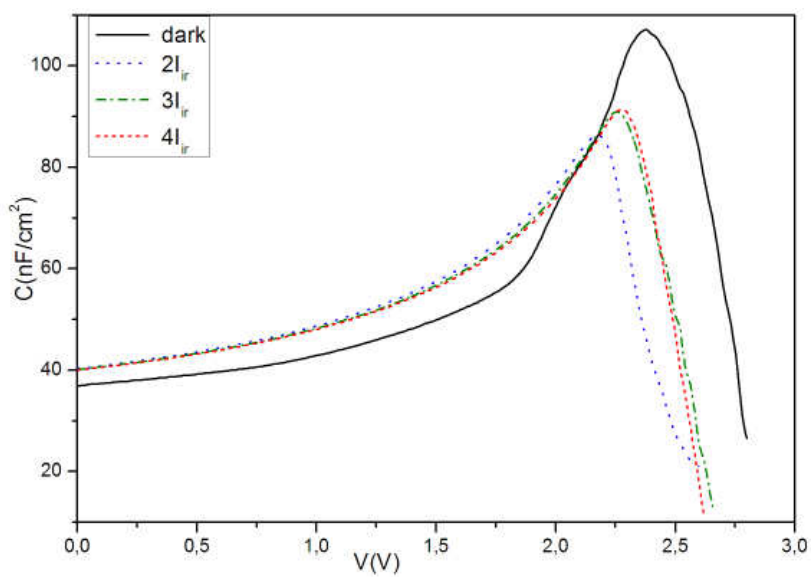

Figure 2. CV curves for a DJ solar cell under infrared illumination $(830 \mathrm{~nm})$ for different laser intensities. 
As on the previous case, the size and form of the capacitance curve vary as the light intensity increases. Here, the peak maximum also shifts to smaller biases and the capacitance value at the equilibrium is larger for the illuminated curves than for the one taken in the dark. However in this case, the capacitance at $V r=0$ remains constant independently of the intensity of the incident light. In fact, the form of the curve does not change so importantly on this occasion, being the shift the most noticeable variation.

Also we can compare the form of the saturated curve obtained under infrared illumination in figure 2 with the one obtained on a previous work for a GalnP diode [11] and we find that they are very similar.

As for the GaAs subcell, we can calculate de doping density from this curve and we find that $N_{\text {GalnP }}=2 \cdot 10^{17} \mathrm{~cm}^{-3}$, which is also the value reported elsewhere [12]

\section{Doping profile on subcells}

After the initial measurements confirmed the validity of the model, we measured some cells and calculated the doping densities and SCR for each subcell. Results are summarized on Table 1. As a remark, on cell 3 we were not able to separate de GaAs cell, as the bandgap energy of the GalnP was bigger than the laser energy, so the cell was not excited and thus, no saturation of the junction was obtained.

\begin{tabular}{ccccc}
\hline Cell ID & $\begin{array}{c}\mathrm{N}_{\text {GaAs }} \\
\left(\mathrm{cm}^{-3}\right)\end{array}$ & $\begin{array}{c}\mathrm{SCR}_{\text {GaAs }} \\
(\mathrm{nm})\end{array}$ & $\begin{array}{c}\mathrm{N}_{\text {GalnP }} \\
\left(\mathrm{cm}^{-3}\right)\end{array}$ & $\begin{array}{c}\mathrm{SCR}_{\text {GalnP }} \\
(\mathrm{nm})\end{array}$ \\
\hline Cell 1 & $1.4 \cdot 10^{1 /}$ & 290 & $2.3 \cdot 10^{1 /}$ & 220 \\
Cell 2 & $\begin{array}{c}1.05 \cdot 10^{17} \\
*\end{array}$ & $\begin{array}{c}* \\
*\end{array}$ & $2.1 \cdot 10^{17}$ & 240 \\
Cell 3 & & $1.7 \cdot 10^{17}$ & 280
\end{tabular}

Table 1: Values obtained for the different measured cells

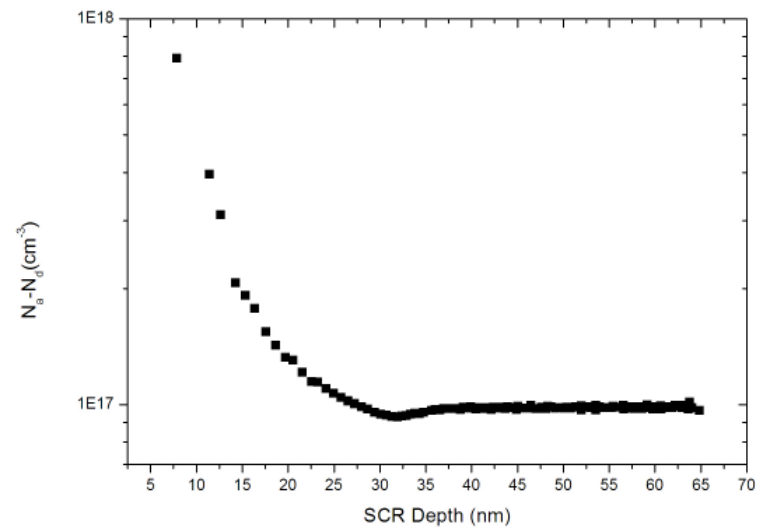

Figure 3. Doping profile for the GaAs subcell on cell 1

Also, doping profiles were calculated for both subcells for the Cell 1 and Cell2 from the saturated curves. On Figure 3 the doping profile for the GaAs subcell of Cell 1 is plotted. Cell 2 profile was very similar, so it is not plotted in sake of clarity.

The plot corresponds to what can be expected for an abrupt homojunction. Absolute doping values are slightly different from the ones presented on table 1, but it enters within the limits of error. It is safe to assume that the final CV curve represents the GaAs subcell.

On figure 4 the profiles of the GalnP subcell for cells 1 and 2 are plotted. Unlike the GaAs, on this case the two profiles are different, both in shape and in magnitude. These differences are not expected as the performance of both cells is very similar. The difference can be related to a partial absortion of the $854 \mathrm{~nm}$ light by the GalnP layers that would lead to a partial increase of the acceptor density. This absortion can be due to either to a different thickness of the GalnP absorber layer on each cell, or to a lower band gap for the cell 2 GalnP subcell that would change not only the absortion of the material but also the dielectric permittivity that is used for the calculation of the profile.

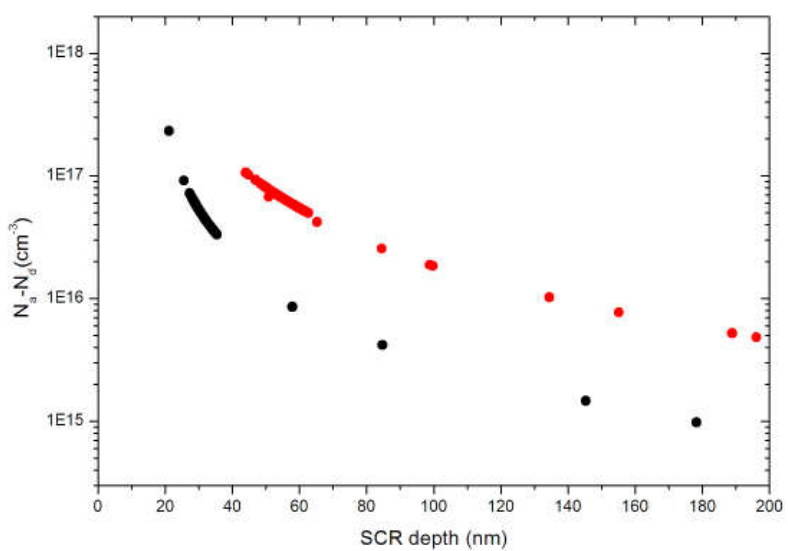

Figure 4. Doping profile for the GalnP subcells on cell 1 (black dots) and cell 2 (red dots)

In any case, the profiles for both subcells are also within the range of the expected values for these kind of diodes.

\section{CONCLUSIONS}

On this work we propose a method for measuring Capacitance-Voltage curves on multijunction solar cells for the analysis of independent subcells. Measurements have been carried on double and triple junctions III-V solar cells and results have been discussed.

We have demonstrated the possibility of isolating a subcell on a multijunction stack by illuminating the rest of the stack with near bandgap energy excitation. The calculations of the carrier density for each case show an agreement with the expected values.Also, doping profiles for GaAs and GalnP subcells have been calculated form 
the CV saturated curves. All this calculations are coherent with the fabricated devices, validating our model.

All these results are the initial stage for developing further analysis on III-V multijunctions based on capacitance measurements such as admittance and DLCP.

\section{REFERENCES}

[1]J.Burdick, T.Glatfelter, Solar Cells 18, 1986, 301-314

[2] G.F. Virshup, Proceedings of the 21st IEEE Photovoltaic Specialist Conference, 1990, Kissimimee, 1249-1255

[3] M. Meusel, C. Baur, G. Letay, A.W. Bett, W. Warta and E. Fernandez, Prog. Photovolt: Res. Appl 11, 2003, 494514

[4]D. L. King, B. R. Hansen, J. M. Moore, and D. J. Aiken, Proceedings of the 28th IEEE Photovoltaic Specialists Conference, 2000, 1197-1201.

[5]Thomas Kirchartz, Uwe Rau, Martin Hermle, Andreas W. Bett, Anke Helbig, and Jürgen H. Werner, Appl. Phys. Lett. 92, 2008,123502

[6] U. Rau, Phys. Rev. B 76, 2007, 085303

[7]T. Walter, R. Herberholz, C. Müller, and H. W. Schock J. Appl. Phys. 80 , 1996, 4411

[8] M. Igalson, C. Platzer-Björkman, Solar Energy Materials and Solar Cells 84,2004, 93

[9] M.Igalson, M.Cwil, M.Edoff, Thin Solid Films 515, 2007, 6142

[10] Jennifer T. Heath, J. David Cohen, William N.Shafarman, J. Appl. Phys, 95, 2004, 1

[11] Carmen M. Ruiz, Ignacio Rey-Stolle, Iván García, Enrique Barrigón, Pilar Espinet, Edgardo Saucedo, Verónica Bermúdez, Carlos Algora Proceedings of the 34th IEEE Photovoltaics Specialist Conference,2009. 1601-1604

[12] I. García, I. Rey-Stolle, B. Galiana, and C. Algora, Appl. Phys. Lett. 94, 2009, 053509

[13]E. Barrigón, I. Rey-Stolle, B. Galiana , I. Garcia, C. Algora Proc. of the 7th Spanish Conference on Electron Devices, IEEE, 2009 Santiago de Compostela, Spain
[14] S. M. Sze in "Semiconductor Devices: Physics and Technology", John Wiley and Sons, 2. Ed. USA 2003 